GA-A23986

\title{
MODELING OF COUPLED EDGE STOCHASTIC AND CORE RESONANT MAGNETIC FIELD EFFECTS IN DIVERTED TOKAMAKS
}

\author{
by \\ T.E. EVANS and R.A. MOYER
}




\section{DISCLAIMER}

This report was prepared as an account of work sponsored by an agency of the United States Government. Neither the United States Government nor any agency thereof, nor any of their employees, makes any warranty, express or implied, or assumes any legal liability or responsibility for the accuracy, completeness, or usefulness of any information, apparatus, product, or process disclosed, or represents that its use would not infringe privately owned rights. Reference herein to any specific commercial product, process, or service by trade name, trademark, manufacturer, or otherwise, does not necessarily constitute or imply its endorsement, recommendation, or favoring by the United States Government or any agency thereof. The views and opinions of authors expressed herein do not necessarily state or reflect those of the United States Government or any agency thereof. 


\title{
MODELING OF COUPLED EDGE STOCHASTIC AND CORE RESONANT MAGNETIC FIELD EFFECTS IN DIVERTED TOKAMAKS
}

\author{
by \\ T.E. EVANS and R.A. MOYER ${ }^{\dagger}$ \\ tUniversity of California, San Diego
}

This is a preprint of a paper presented at the 15th International Conference on Plasma Surface Interactions in Controlled Fusion Devices, May 27-31, 2002, in Gifu, Japan, and to be published in the Proceedings.

\author{
Work supported by \\ the U.S. Department of Energy \\ under Contract DE-AC03-99ER54463 \\ and Grant DE-FG03-95ER54294
}




\begin{abstract}
Attaining the highest performance in poloidally diverted tokamaks requires resonant magnetic perturbation coils to avoid core instabilities (locked, resistive wall and neoclassical tearing modes). These coils also perturb the pedestal and edge region, causing varying degrees of stochasticity with remnant islands. The effects of the DIII-D locked mode control coil on the edge and core of Ohmic plasmas are modeled with the field line integration code TRIP3D and compared with experimental measurements. Without detailed profile analysis and field line integration, it is difficult to establish whether a given response is due to a "core mode" or an "edge stochastic boundary." In diverted Ohmic plasmas, the boundary stochastic layer displays many characteristics associated with such layers in non-diverted tokamaks. Comparison with stochastic boundary results from non-diverted tokamaks indicates that a significant difference in diverted tokamaks is a "focusing" of the magnetic field line loss into the vicinity of the divertor.
\end{abstract}




\section{INTRODUCTION}

The ultimate success of the tokamak concept depends critically on the maximum level of performance that can be attained. Performance in poloidally diverted tokamaks is typically optimized using resonant magnetic perturbation coils to avoid core instabilities such as locked, resistive wall, and neoclassical tearing modes [1-3] which limit the attainable plasma pressure, reduce energy confinement, and can even lead to disruptions. Although designed to control core modes, these coils also perturb the pedestal and scrape-off layer. These boundary perturbations can result in stochastic layers that alter plasma-wall interactions. Because the stochastic layer forms near the unperturbed seperatrix, it can also impact core performance due to the tight coupling of the core transport to pedestal plasma conditions [4].

Our previous field line modeling work focused on calculating the scaling of the stochastic flux loss in the DIII-D tokamak with a singe C-coil phase [5]. These results provided quantitative estimates of how much magnetic flux connects to plasma facing surfaces, from inside the unperturbed seperatrix, as a function of divertor configuration, the edge magnetic shear, and the amplitude of the current in the external control coils, using the TRIP3D field line integration code. The TRIP3D code uses high resolution, double precision, EFIT Grad-Shafranov equilibria reconstructions [6] that are constrained by experimental data from the DIII-D flux loops and magnetic probes and measured profiles. These EFIT equilbria specify the axisymmetric background fields in TRIP3D. We then superimpose non-axisymmetric perturbation fields from a discrete line filament model of the individual C-coil loops using a Biot-Savart law algorithm. At the highest C-coil currents, it was found that as much as $25 \%$ of the poloidal magnetic flux connects the pedestal region to various plasma facing surfaces.

In this paper we look at the core and edge plasma response to changes in C-coil current amplitudes and phases. We then model changes in the 3-D vacuum magnetic field topology and compare the modeling results to the measured plasma response. We find that the core and boundary responses are tightly coupled, and that it can often be difficult to determine empirically which effects are "core effects" (e.g., locked modes) versus which effects result from the formation of a stochastic boundary. 


\section{CORE AND EDGE PLASMA EFFECTS PRODUCED BY THE DIII-D C-COIL}

The DIII-D C-coil primarily drives $\mathrm{n}=1$ modes with an $\mathrm{m}=1$ component that is about twice as large as the $\mathrm{m}=2$ mode (here, $\mathrm{n}$ and $\mathrm{m}$ are respectively the toroidal and poloidal mode numbers). It also contains significant $\mathrm{m}=3-6$ harmonics, and consequently directly perturbs both the core and edge plasma. In this section we provide an example of the difficulties encountered when interpreting the plasma response to the $\mathrm{C}$-coil.

We consider two double-null diverted Ohmic plasmas with the ion $\nabla \mathrm{B}$ drift direction pointed up. During the first $2500 \mathrm{~ms}$ of these discharges the C-coil is actively controlled using our standard locked mode feedback algorithm. At $2500 \mathrm{~ms}$ the amplitude and/or phase of the six coil segments are changed over a $100 \mathrm{~ms}$ timescale. Figure 1 shows how the line average density $\mathrm{n}_{\mathrm{e}}$ (a) and lower divertor $\mathrm{D}_{\alpha}$ recycling (e) change with the C-coil currents (b) in discharge 110546. The three opposing loops to those in (b) are hardwired for equal but opposite currents. Locked mode amplitude and phase signals (c,d) are also shown.

During this discharge $\mathrm{n}_{\mathrm{e}}$ drops dramatically and the lower divertor recycling starts to increase after the $\mathrm{C}$-coil currents are changed but the discharge then transitions into a bursty or chaotic looking behavior. The locked mode signals change somewhat during the C-coil transient then assume new values.

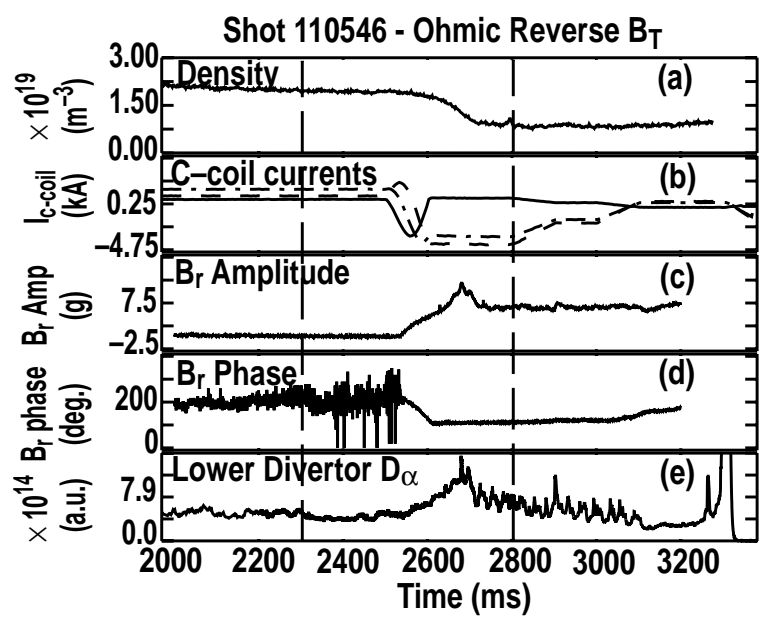

Fig. 1. Plasma response to a C-coil change during discharge 110546: (a) line average density, (b) C-coil currents, (c) uncompensated "locked mode detector" signals pr12 \& pr13, (d) compensated "locked mode detector" signals esld079, esld139, and esld199, and (e) lower divertor $D_{\alpha}$ recycling fs04f near the outer strikepoiont.

In Fig. 2, discharge 110544 begins with the same plasma as in Fig. 1 but the programmed C-coil currents (b) after $2500 \mathrm{~ms}$ are somewhat different. Note that the $\mathrm{n}_{\mathrm{e}}$ response (a) and lower 


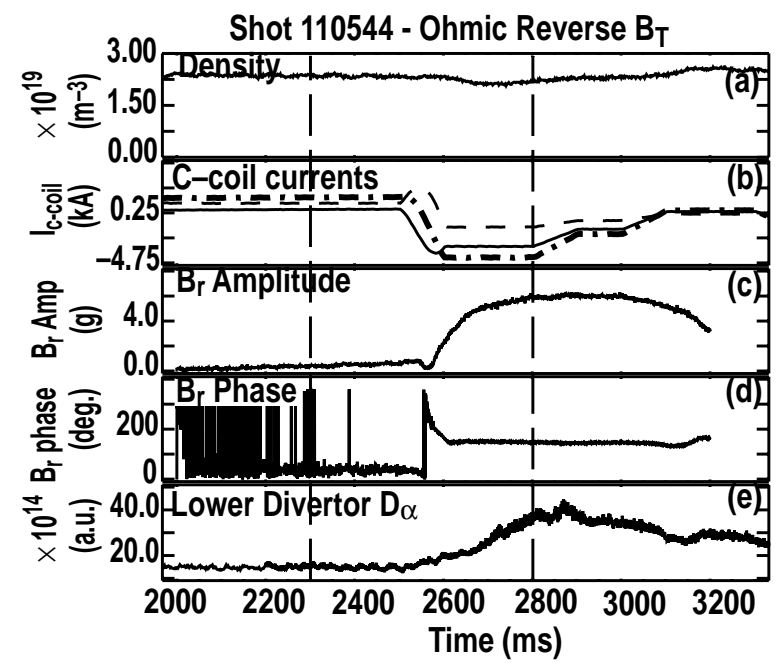

Fig.. 2. Plasma response to a C-coil change during discharge 110544: (a) line average density, (b) C-coil currents, (c) uncompensated "locked mode detector" signals pr12 \& prl3, (d) compensated "locked mode detector" signals esld079, esld139, and esld199, and (e) lower divertor $D_{\alpha}$ recycling $f_{s} 04 f$ near the outer strikepoiont.

divertor recycling (e) as well as some of the locked mode signals (c) and (d) differ significantly from those in Fig. 1.

This discharge had a small transient $82.7 \mathrm{~T}-\ell / \mathrm{s}$ peak deuterium gas puff ( 100 ms FWHM) starting at $2600 \mathrm{~ms}$ (about 1/2 the usual plasma startup fueling rate) that was not present in shot 110546. This puff may have changed the recycling response somewhat but does not appear to explain the very different core and edge responses seen in these two cases. During shot 110544, $\mathrm{n}_{\mathrm{e}}$ and the stored energy initially drop but then recover and continue to increase after the small gas puff (ending at $2875 \mathrm{~ms}$ ) although the recycling is decreasing and the locked mode signals are constant. This behavior suggests that the confinement is improving during this phase, which is inconsistent with the characteristics typical of locked modes.

In order to determine if these responses are due to core (locked mode) or boundary effects, we examine the $n_{e}$ and $T_{e}$ profiles measured by Thomson scattering. Figure 3 shows full $n_{e}$ (a) and $\mathrm{T}_{\mathrm{e}}$ (b) profiles, averaged over $100 \mathrm{~ms}$ (six sample times), before (2300 ms) and after (2800 ms) the C-coil current changes in discharges 110546 and 110544.

In 110544 (circles) there is no change when the c-coil currents change in $n_{e}$ or $T_{e}$ near the $q=2$ surface where locked modes are expected to have the largest effect in DIII-D. There is a substantial increase in $\mathrm{n}_{\mathrm{e}}$ outside the $q=3$ surface, and there are changes in $\mathrm{T}_{\mathrm{e}}$ of limited radial extent when the coil current is changed. In contrast, during 110546, the profiles at each current are dramatically different starting just inside the $q=4$ surface extending almost to the center. Both the $\mathrm{n}_{\mathrm{e}}$ and $\mathrm{T}_{\mathrm{e}}$ profiles drop substantially from the edge to well inside the $q=2$ surface and the confinement is globally degraded. The edge profiles in these discharges, [Fig. 3(c,d)], also differ. In $110546 \mathrm{n}_{\mathrm{e}}$ is unchanged outside $q=4$ while $\mathrm{T}_{\mathrm{e}}$ is reduced across the edge. In $110544 \mathrm{n}_{\mathrm{e}}$ 

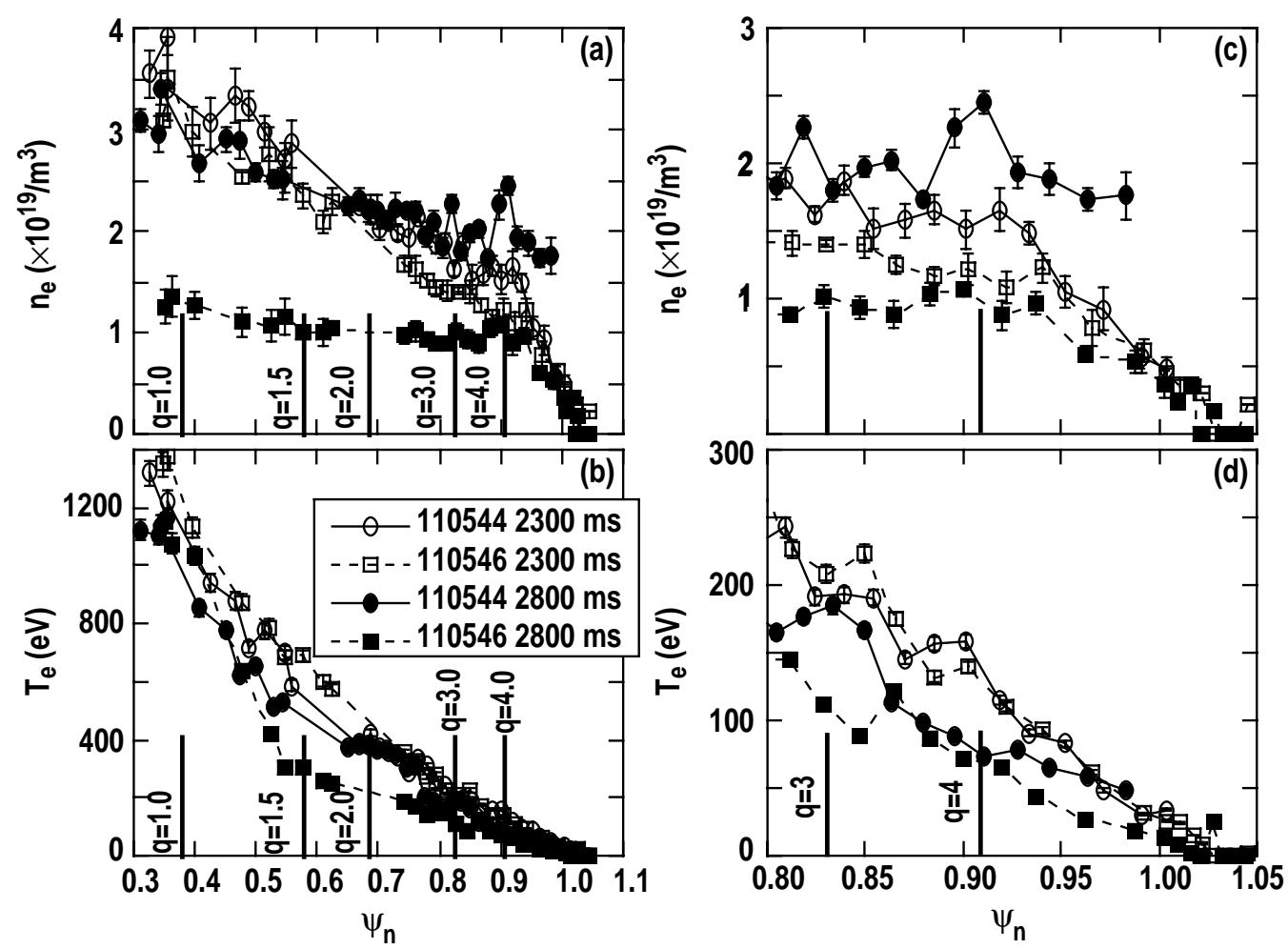

Fig. 3. Full (a) $n_{e}$ and (b) $T_{e}$ and edge (c) $n_{e}$ and (d) $T_{e}$ Thomson profiles for the discharges shown in Figs. 1 and 2 at 2300 and $2800 \mathrm{~ms}$. The profiles are averaged over $100 \mathrm{~ms}$ (6 Thomson time samples). The locations of the qsurfaces in the axisymmetric equilibria are indicated.as vertical lines.

increases substantially over the 9 outermost Thomson points (covering the $q=3,4$ and 5 surfaces) and $\mathrm{T}_{\mathrm{e}}$ drops across the same 9 channels.

In discharge 110544, the recycling not only increases (Fig. 2) but broadens across the lower divertor floor. Figure 4 shows the change in normalized $\mathrm{D}_{\alpha}$ recycling from four chords viewing the lower outer strike point at $2300 \mathrm{~ms}$ (shaded) and $2800 \mathrm{~ms}$ (white). Each bar represents the local recycling measured by a single chord divided by the total recycling from all four chords (inset). Each chord is referenced to the outer strike point radius ( $\delta \mathrm{R}=$ outer strike point major radius $R_{s p}$ minus the $D_{\alpha}$ view major radius $R_{D \alpha}$ ). The recycling profile is broadened, consistent with field line spreading near the strike point while the increase in the overall level of recycling implies that more particles are flowing into the divertor region. 


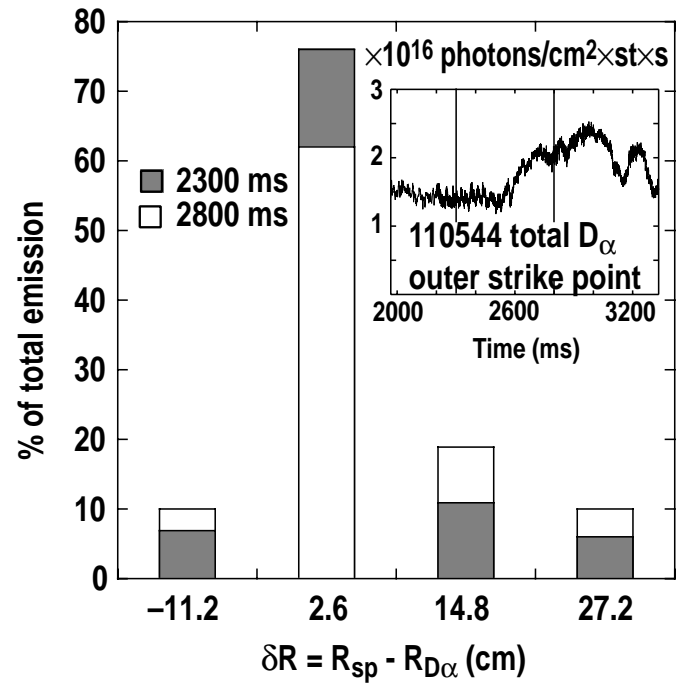

Fig. 4. Change in the normalized $D_{\alpha}$ recycling at four locations near the outer strike point (located at dr $\left.=0\right)$ for two C-coil currents, one at $2300 \mathrm{~ms}$ and the other at $2800 \mathrm{~ms}$. Here normalized recycling is the recycling at a point divided by the total recycling integrated across each of the four points (shown as a function of time in the insert). 


\section{FIELD LINE INTEGRATION AND MAGNETIC STRUCTURE IN DIII-D}

The magnetic field line integration code TRIP3D is used to study the pedestal, separatrix and SOL topology of DIII-D. TRIP3D was adapted to DIII-D [5] from a previous code, TRIPND, developed to study stochastic layers in low elongation wall limited tokamaks such as Tore Supra [7]. We use the code to model shot 110544 at $2800 \mathrm{~ms}$ and $2300 \mathrm{~ms}$ (see the dashed lines in Fig. 2).

At $2800 \mathrm{~ms}$ the unperturbed outer seperatrix position from the EFIT solution is located at $\mathrm{r}_{\text {sep_mid_out }}=0.6349 \mathrm{~m}$ [Fig. 5(a)]. The solid violet line is the DIII-D vessel wall and the dashed blue line is the unperturbed EFIT separatrix position. The figure shows the field line r, $\theta$ positions after each toroidal transit (black, red and green dots) at a toroidal angle $\phi=120^{\circ}$ (the Thomson scattering system location: dark blue dots near $\theta=60^{\circ}$ ). The field lines are started at $\phi=120^{\circ}, \theta=0$

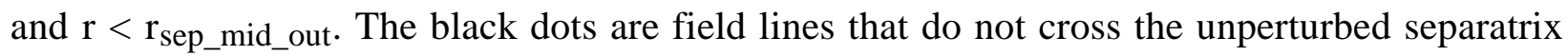
while the red and green dots are those field lines that cross the separatrix and intersect a material surface. Red field lines are integrated in the forward $\mathrm{B}_{\mathrm{T}}$ direction and green lines are integrated in the reverse direction.
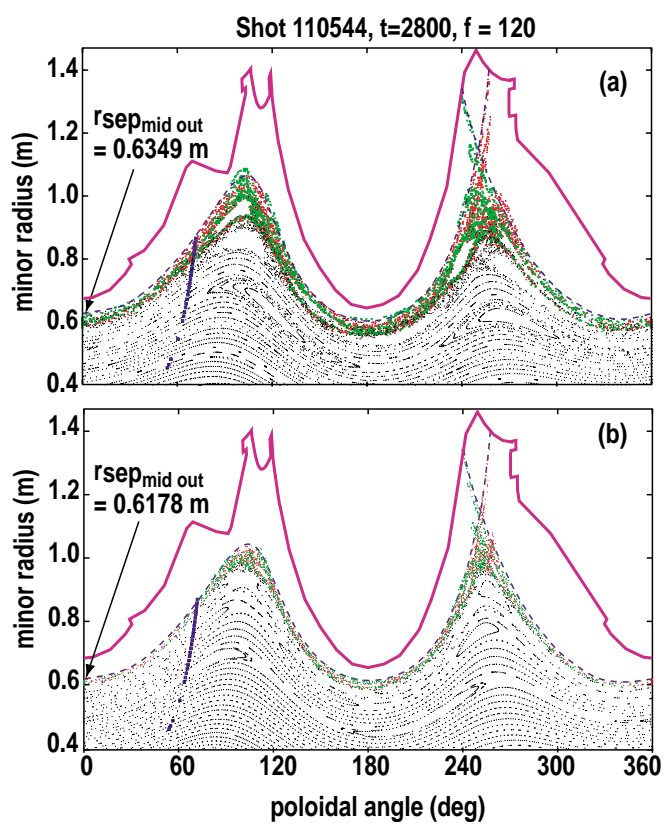

Fig. 5. Pedestal field line modeling results from the TRIP3D code for DIII-D shot 110544 at (a) 2800 ms and (b) $2300 \mathrm{~ms}$. Black dots represent field lines that do not cross the unperturbed EFIT separatrix. Red and green dots represent field lines integrated in the forward (red) and reverse (green).BT direction that intersect a material surface. 
The modeling results demonstrate that the C-coil currents and phases at $2800 \mathrm{~ms}$ produce a much broader open field line stochastic region (OFLSR) than those at $2300 \mathrm{~ms}$. We find that the width of the OFLSR increases by about a factor of 2.5 at the outer midplane between 2300 and $2800 \mathrm{~ms}$. Field lines were initiated at $0.5 \mathrm{~mm}$ steps starting about $50 \mathrm{~mm}$ inside the unperturbed separatrix for each timeslice. Based on the starting radius of the first field line connecting to a material surface we determine the width of the OFLSR. At $2300 \mathrm{~ms}$ the width of the OFLSR is $19 \mathrm{~mm}$ and at $2800 \mathrm{~ms}$ it is $44 \mathrm{~mm}$. This implies that the number of Thomson scattering points sampling the OFLSR increases from 3 at $2300 \mathrm{~ms}$ to 9 at $2800 \mathrm{~ms}$.

The structure of the field lines near the lower divertor $\mathrm{X}$-point indicates that the footprint around the strikepoints is more complex at 2800 than at $2300 \mathrm{~ms}$. In addition to those field lines lost very close to the unperturbed inner and outer divertor legs we see secondary striations further away from the legs at $2800 \mathrm{~ms}$ that are not seen at $2300 \mathrm{~ms}$. These secondary striations intersect the divertor about 100-135 mm away from the nominal strikepoints (both along the inner wall above the inner strikepoint and outside the outer stikepoint in the outer scrape-off layer). These secondary striations indicate a significant broadening of the magnetic flux profiles on the divertor targets and thus a broadening of the heat and particle flux profiles. 


\section{DISCUSSION AND CONCLUSIONS}

In DIII-D, locked mode control coils perturb both the core and edge depending upon the coil currents, phasing, and plasma configuration. The core perturbation globally reduces confinement by destabilizing modes near the $q=2$ surface (in 110546 a locked mode). The edge perturbation is consistent with formation of a stochastic boundary layer with characteristics associated with such layers in non-diverted tokamaks: 1) $\mathrm{T}_{\mathrm{e}}$ and $\mathrm{n}_{\mathrm{e}}$ profile flattening locally in the edge; 2) increased recycling consistent with connecting field lines from inside the separatrix to the divertor; and 3) broadened particle flux profile on the divertor floor. Magnetic field line integration using TRIP3D predicts that the width of the open field line stochastic region along the Thomson chord increases by about a factor of 2.5 when the C-coil currents change in 110544 , consistent with the increased flattening of the edge profiles. The predicted field line spreading in the lower divertor is in agreement with the recycling profile broadening in the divertor. These results suggest that for Ohmic plasmas the nonlinear plasma response may be insignificant. These similarities may not hold at higher heating powers and momentum input, although in some high performance discharges these features are predicted and seen [5]. Comparison of these results with stochastic boundary results from non-diverted tokamaks indicates that a significant difference in diverted tokamaks is a "focusing" of the magnetic field line loss into the vicinity of the divertor

Without detailed profile analysis and field line integration, it is difficult to establish whether a given response is a "core mode" or an "edge stochastic boundary". We have shown that the standard locked mode detector signals are not always indicative of a significant plasma response at the $q=2$ surface. In some cases, e.g. (110544) a locked mode detector response is induced when the dominant magnetic perturbations are in the edge magnetic topology. We have identified a specific case in which changes in the $\mathrm{C}$-coil currents produce a broadening of the $\mathrm{D}_{\alpha}$ recycling profile in the lower divertor and a flattening of the edge $T_{e}$ and $n_{e}$ profiles without changing these profiles at the $q=2$ surface. However, in another case (110546) C-coil charges of similar magnitude clearly induce a locked mode with the usual negative effects on confinement.

We conclude that specialized, high poloidal mode number coils, are required for employing stochasticity to control the pedestal without directly perturbing the core plasma. Most likely, independent coil sets will be required to control core modes and to control pedestal transport. 


\section{REFERENCES}

[1] R.J. La Haye, A.W. Hyatt, S.T. Scoville, Nucl. Fusion 32, (1992) 2119.

[2] A.M. Garofalo, T.H. Jensen, L.C. Johnson, et al., Phys. Plasmas 9 (2002) 1997.

[3] R.J. La Haye, S. Gunter, D.A. Humphreys, J. Lohr, T.C. Luce, M.E. Maraschek, C.C. Petty, R. Prater, J.T. Scoville, E.J. Strait, Phys. Plasmas 9 (2002) 2051.

[4] T.H. Osborne, et al., Phys. Plasmas 8, (2002) 2022.

[5] T.E. Evans, R. A. Moyer, and P. Monat, "Modeling of stochastic magnetic flux loss from the edge of a poloidally diverted tokamak," General Atomics Report GA-A23965, May 2002, submitted to Phys. of Plasmas.

[6] L.L. Lao, H.E. St. John, R.D. Stambaugh, et al., Nucl. Fusion 25 (1985) 1611.

[7] T.E. Evans, Proc. 18th European Physical Society Conference on Controlled Fusion and Plasma Physics, Berlin, 1991 (European Physical Society, Petit-Lancy, 1991) Part II, p. 65. 


\section{ACKNOWLEDGMENT}

Work supported by U.S. Department of Energy under Contract DE-AC03-99ER54463 and Grant DE-FG03-95ER54294. 